\title{
Sequential sampling of surface-mined land to assess reclamation
}

\author{
MICHAEI AMES
}

\begin{abstract}
Author is assistant professor, Department of Experimental Statistics. College of Agriculture and Home Economics, New Mexico State University, Las Cruces 88003.
\end{abstract}

\begin{abstract}
Yearly, a larger percentage of government regulations are stated in statistical terms. More than simple techniques are sometimes necessary to comply with these regulations, both in analyzing and designing experiments and surveys. The problem considered in this paper is how to efficiently sample a mining site to show that the site has been successfully reclaimed. Different sequential analyses are suggested and a two-stage plan by Stein is recommended and illustrated. This approach allows us to sample with a predetermined probability of detecting a successful reclamation. It is pointed out that the usual sample size formulas are not directly applicable to this problem.
\end{abstract}

Key Words: reclaimed land, repeated significance tests, sample size, sequential analysis, sequential $t$-tests

According to the Federal Register (Vol. 44, No. 50, 13 March 1979), requirements for proving successful reclamation of surfacemined lands include: "the ground cover and productivity of the revegetated area shall be considered equal if they are at least $90 \%$ of the ground cover and productivity of the reference area with $90 \%$ statistical confidence." Suppose, $\mathbf{x}_{1}, \mathbf{x}_{2}, \ldots, \mathbf{x}_{\mathbf{k}}, \ldots$ are the proportions, say from a standard 10-pin point frame, of cover from sample locations in a reclaimed area, with mean $\mu$ and variance $\sigma^{2}$. Suppose, also, that $\mu_{.9}=.9 \mu_{\mathrm{r}}$, is $90 \%$ of the mean cover of the reference area. The requirements seem to say that successfully documenting a reclaimed area requires being able to reject the null hypothesis

in favor of $\begin{aligned} \mathrm{H}_{0}: \mu<\mu .9 \\ \mathrm{Ha}: \mu>\mu .9\end{aligned}$

with no more than a $10 \%$ Type I error rate.

For a fixed sample size this is a straight forward one-sided $t$-test, which rejects $H_{0}$ if, for a sample of $k$,

$$
\frac{\left(\bar{x}_{\mathrm{k}}-\mu .9\right)}{s_{\mathrm{k}} / \sqrt{k}}>t_{\mathrm{k}-1,90}
$$

Where $t_{\mathrm{k}-1,90}$ is the $90 \%$ percentile of the $t$-distribution with $\mathrm{k}-1$ degrees of freedom. Part of designing the survey is determining what sample size to use. The sample size in a test of hypothesis determines the probability that the alternative will be accepted when it is true, in other words the power of the test. We want to find a sample size with which there is a high probability of detecting a successful reclamation, that is rejecting $\mathrm{H}_{0}$ if it is false.

This is not a problem of parameter estimation. Suppose it were desired to estimate $\mu-\mu_{. \theta}$ to within plus or minus $\mathrm{E}$. Then the required sample size is

$$
n=(z \sigma)^{2} / E^{2}
$$

where $z$ (a percentile of the standard normal distribution) and $E$ are

This work was supported by the NMSU Agricultural Experiment Station. sometimes replaced by a t-percentile and an estimate of the variance, $s$. This is the approach taken by Hofmann and Reis (1990) and Chambers and Brown (1983) in determining sample size. The idea is to take $E$ to be some very small number, say (following Chambers and Brown) one-fourth or one-half of $1 \%$ of the mean of the reference area.

This is the right answer but to the wrong question (Ortiz and Ames 1992). The goal is not to estimate $\mu-\mu .9$ but to prove that $\mu$ is greater than $\mu .9$. If $\mu$ is much larger than $\mu .9$ then the sample size found in equation 1 is much more than is needed, whereas for $\mu$ barely larger than $\mu, 9$ a larger sample is needed. And if $\mu$ is less than $\mu .9$ there is no sample size that will let us prove otherwise. In this case, the sample size is determined by the desired power (Steele and Torrie 1960), not by how closely we wish to estimate the parameter. Power can be found before the sample is taken if the variance is known. If not known, it is necessary to either take the largest sample size affordable to get the most power or sample sequentially, meaning take 1 or more small samples and come to a conclusion for or against $\mathrm{H}_{0}$ based on the accumulated evidence. The sequential approach is the subject of this paper. In the next section the sequential sampling problem is outlined. After that, examples of different approaches are given.

\section{Background}

The first complete theoretical development in sequential sampling is probably Wald (1947), although earlier researchers used similar approaches in quality control problems. Even this work, however, assumed that the variance was known. Their goal was to reduce, if possible, the sample size from a fixed sample test for the same power. This is done, basically, by sampling one extra point at a time or a small number of points at a time, recalculating a test statistic each time new data are collected, and reperforming a test. The conclusion, at each step, is to either reject the null, accept the null, or suspend judgment. Unfortunately, it is not as straightforward as recalculating the statistic in equation 1 each time; the type 1 error rate, for one thing, does not stay constant by doing this. It is necessary to modify somehow either the statistic or critical value at each step.

In any sequence of experiments the result is a corresponding sequence of test statistics, a stochastic process. Think of the values of the test statistic wandering around between 2 boundaries as more samples are taken. As soon as one or the other of the boundaries is crossed, a decision can be reached for or against the null hypothesis, assuming a 1 sided null hypothesis. (In a 2 sided null hypothesis, crossing either boundary will reject the null, while going too long without doing so implies that the null is not sufficiently incorrect to worry about). Without going into the theory of stochastic processes and random walks, consider that in equation 1 , for sequential sampling, the sample size is now also a random variable not independent of the values of the sample mean and 
variance. The mathematics involved for the true distribution of such statistics is quite complex. Where this has proven intractable, asymptotic results and ad hoc methods are used instead.

\section{The Sequential Probability Ratio Test}

Because Wald's work on the sequential probability ratio test forms the corner-stone for sequential sampling procedures, a review of this is in order. Consider testing the 2 hypotheses

versus

$$
\begin{aligned}
\mathrm{H}_{1}: \mu & =\mu_{1} \\
\mathrm{H}_{2}: \mu & =\mu_{2}
\end{aligned}
$$

with $\mu_{1}<\mu_{2}$. Let $\alpha$ be the probability of rejecting $H_{1}$ when $\mu=\mu_{1}$ and $\beta$ be the opposite risk. If $f_{1}(x)$ and $f_{2}(x)$ are the probability density functions for the data under $\mathrm{H}_{1}$ and $\mathrm{H}_{2}$, respectively, the likelihood ratio criterion for building tests of hypotheses is to accept $H_{1}$ if $f_{1}(x) / f_{2}(x)>\lambda_{1}$ and accept $H_{2}$ if $f_{1}(x) / f_{2}(x)<\lambda_{2}$. The rationale is that because the distribution measures the probability of an outcome, large $f_{1}(x) / f_{2}(x)$ implies that there is a greater chance that the data came from the first population than the second, and vice versa if the ratio is small. $\lambda_{1}$ and $\lambda_{2}$ are determined by $\alpha$ and $\beta$. Wald's sequential probability ratio test is to sample as long as

$$
\left.\log _{e}(\beta / 1-\alpha)\right)<-\left\{\Sigma\left(x_{i} \neq \mu_{2}\right)^{2}-\Sigma\left(x_{i}-\mu_{1}\right)^{2}\right\} / 2 \sigma^{2}<\log _{e}(1-\beta) / \alpha
$$

which, unfortunately, is a function of the unknown variance.

Weiss and Wolfowitz (1972) suggest taking a large enough preliminary sample to get a good estimate of $\sigma^{2}$ and then proceeding as though this were the true variance. They show that for "small" differences between $\mu_{1}$ and $\mu_{2}$ this modified sequential probability ratio test is consistent and efficient, meaning it does just about as good $a$ job as it would if the true variance were known. Hall (1962), starting with the same test, modifies the critical regions slightly and proceeds as Weiss and Wolfowitz suggest.

The connection with this to the reclamation problem is we can set $\mu_{1}=\mu_{.9}$ and $\alpha=.10$. The values of $\mu_{2}$ and $\beta$ are specified by the researcher so that he can be at least $1-\beta$ sure of being able to say the site has been reclaimed when the true cover rate is $\mu_{2}$. Even a very small difference between the null and true mean can be detected if the sample is large enough, but it may be necessary to give up the precision necessary to find this difference if the sample size needed is too large. For example, suppose that $90 \%$ of the mean of the reference area is $75 \%$ ground cover. If the mean of the reclaimed area is $76 \%$, then the site has been successfully reclaimed, $\mathrm{H}_{0}$ is false, and there is a sample size that would give us a good chance of being able to reject $\mathbf{H}_{0}$. But the sample would likely be very large.

\section{Other Tests and Stein's Method}

The physical requirements of the reclamation testing problem would suggest that some simple, quick, sampling and analysis methods that can be performed, as much as possible, in the field are to be preferred, say by entering data into a programmable handheld calculator as it is taken. Sequential $t$-tests and multiplesampling plans are such possibilities. The sequential $t$-tests starts with a sample of $k$ and continues samplings, 1 more point at a time, with the sample mean, variance and $t$-statistics recalculated with each new point. There are more than a few sequential $t$-tests proposed in the literature, each with slightly different properties. For example, a plan outlined in Siegmund (1985, p. 116-121) allows for a predetermined stopping point beyond which sampling stops if a decision has not been reached. This is an example of a "closed" plan (Armitage 1957 and Schneiderman and Armitage 1962). An "open" plan (National Bureau of Standards 1951) is one that has the theoretical possibility of never reaching a conclusion. Readers can start with the recent monograms of Siegmund (1985) and Wetherill and Glazebrook (1986) and work backwards for an overview of the literature on sequential $t$-tests.

Stein (1945) proposed the following two-stage plan has some advantages over the sequential $t$-test, mostly in simplicity. Take a sample of $k$ observations to get an estimate of $\mu$ and $\sigma^{2}$, namely $\bar{x}_{k}$ and $\mathrm{s}_{\mathbf{k}}^{2}$. If $\mathrm{H}_{\mathrm{o}}$ or $\mathrm{H}_{\mathrm{a}}$ can be rejected based on this sample, then stop. If not, take $n_{0}=\left[s_{k}^{2} / w\right]+1-k$ more observations ([.] is the greatest integer function) with $w$ chosen to give the test a specified power (see below). From the entire sample calculate the sample average, $\bar{x}_{\mathrm{n}}$, and reject $\mathrm{H}_{\mathrm{o}}$ if

$$
\frac{\left(\overline{x_{\mathrm{n}}}-\mu .8\right)}{S_{\mathrm{k}} / \sqrt{n}}>\mathrm{t}_{\mathrm{k}-1,10}
$$

Stein shows that a lower bound for the power of this test is

$$
1-\beta=P \quad\left[\mathbf{t}_{k-1}>t_{k-1, .90}-\frac{\mu-\mu_{.9}}{\sqrt{w}}\right]
$$

where $t_{\mathrm{k}-1}$ is a $t$ random variable with $\mathrm{k}-1$ degrees of freedom. This can be computed from a good set of $t$ tables, main-frame packages, or even some hand-held calculators. Equation 4 is used to pick w.

Example. To illustrate, suppose the mean cover at the reference is $83 \%$. Then $90 \%$ of the reference cover is $75 \%$, and suppose further that a test is desired that will have at least a (1$\beta)^{*} 100 \%=50 \%$ chance of rejecting $\mathrm{H}_{0}: \mu<.75$ when $\mu$ is as large as $80 \%$. By changing $\mathrm{k}$ and $\mathrm{w}$ in equation 4 there are many plans that will give these characteristics. Basically, $k$ (the initial sample size) should be as large as possible, say $\mathbf{3 0}$ or more depending on how nearly normal the data are. With $k=30$, w must be chosen so that

$$
.50=P\left\{t_{29}>2.46-.05 / \sqrt{w}\right\}
$$

and $\mathrm{w}=.0004$, because the probability that a $t$ with 29 degrees of freedom is larger than $2.46-.05 / \sqrt{.0004}=0.0$ is about $50 \%$. After the initial sample of 30 is taken, the sample standard deviation is computed and plugged into [ $\left.\mathrm{s}_{30}^{2} / .0004\right]+1-30$. If this is positive, then this many more observations are taken. If not, then the test of hypothesis is performed with the data on hand. Notice that more power requires a smaller $w$ value; but the smaller $w$ is, the larger the second sample will need to be.

\section{Conclusion}

The temptation is, because $n=(z s)^{2} / E^{2}$ is the formula most associated with sample size, to attempt to make all problems fit this result. There is some difference, however, between testing hypotheses and estimating parameters when it comes to determining appropriate sample sizes. This paper provides a critique of this problem and suggests alternative sampling procedures. Specifically, it reviews a class of methods, sequential sampling, that deserves to be better known and more widely used.

\section{Literature Cited}

Armitage, 1957. Restricted sequential procedures. Biometrika, 44:9-26.

Chambers, J.C., and R.W. Brown. 1983. Methods for vegetation sampling and analysis on revegetated mined lands. Tech. Rep. INT-151. Ogden, Ut. USDA Forest Serv.

Hall, W.J. 1962. Some sequential analogs of Stein's two-stage test. Biometrika, 49:367-378.

Hofmann, L., and R.E. Ries. 1990. An evaluation of sample adequacy for point analysis of ground cover. J. Range Manage. 43:545-549.

Ortiz, M., and M. Ames. 1992. Viewpoint: Sample adequacy for point analysis depends on the objectives. J. Range Manage. 45:595.

National Bureau of Standards. 1951. Tables to facilitate sequential t-tests. Applied Mathematics Series, No. 7. U.S. Dep. Commerce, Washington, D.C.

Schneiderman, M.A., and P. Armitage. 1962. Closed sequential t-tests. Biometrika, 49:359-366.

Siegmund, D. 1985. Sequential analysis. Springer-Verlag, N.Y. 
Steele, R.G.D., and J.H. Torrie. 1960. Principles and procedures in statistics. McGraw-Hill, N.Y.
Stein, C.J. 1945. A two sample test for a linear hypothesis whose power is independent of the variance. Ann. Math. Stat. 16:243-258.

Wald, A. 1947. Sequential analysis. John Wiley and Sons, N.Y.

Wetherill, G.B., and K.D. Glazebrook. 1986. Sequential methods in statistics. Chapman and Hall, N.Y. 